\title{
TARTU RADIOCARBON DATES XI
}

\section{EVALD ILVES}

Institute of Zoology and Botany, Academy of Sciences

Tartu, Estonian SSR

\section{INTRODUCTION}

This list includes dates of geologic samples measured using a single-channel liquid scintillation ${ }^{14} \mathrm{C}$ counter at the Geochemical and Statistical Laboratory, Tartu, Estonian SSR. Our modern standard is made of benzene enriched in ${ }^{14} \mathrm{C}$ and its activity is checked with NBS oxalic acid standard sample. Dates are given in conventional radiocarbon years, based on the Libby half-life of $5570 \pm 30 \mathrm{yr}$. AD 1950 is the reference year. Errors refer only to $1 \sigma$ standard deviation calculated from count rates involved.

\section{GEOLOGIC SAMPLES}

\section{Estonian SSR}

\section{Haanja series}

The Haanja upland in SE Estonian SSR belongs to an island deposit. Upper strata of basal Devonian deposits are at $100-160 \mathrm{~m}$ asl. Glacial deposits $100-180 \mathrm{~m}(40-50 \mathrm{~m}$ on slopes) overlie the Devonian strata. The present upland was formed at the end of Valdai glaciation during the Haanja (Luuga) glacier retreat stage 13,500-13,200 BP. The Haanja upland consists of a variety of geologic and morphologic characteristics. Dates listed below are of sediments from center of upland, ca $300 \mathrm{~km}^{2}$.

Samples coll 1976 by H Mäemets (Mäetilga) and by E Ilves and H Mäemets 1980 (Karuniidu), 1981 (Tuuljärv), 1983 (Vaskna).

TA-1081. Mäetilga

$3480 \pm 70$

Bryales peat from depth $370-380 \mathrm{~cm}$. Pollen Zone SB1.

TA-1082. Mäetilga

$5670 \pm 100$

Sapropel with admixture of lime pelite from depth $550-560 \mathrm{~cm}$.

TA-1359. Karuniidu

$870 \pm 60$

Fen peat with wood remains from depth $50-60 \mathrm{~cm}$. Pollen Zone SA3.

TA-1396. Karuniidu

$1100 \pm 60$

Fen peat with wood remains from depth $100-110 \mathrm{~cm}$. Pollen Zone SA2.

TA-1397. Karuniidu

$1920 \pm 60$

Fen peat from depth $150-160 \mathrm{~cm}$. Pollen Zone SA1

TA-1398. Karuniidu

$2180 \pm 60$

Fen peat from depth $200-210 \mathrm{~cm}$. Pollen Zone SAl.

TA-1399. Karuniidu

$2080 \pm 60$

Fen peat from depth $250-260 \mathrm{~cm}$. Pollen Zone SA1.

TA-1400. Karuniidu

$2500 \pm 60$

Fen peat from depth $300-310 \mathrm{~cm}$. Beginning of climatic period SA.

TA-1401. Karuniidu

$3000 \pm 60$

Fen peat from depth $350-360 \mathrm{~cm}$. Pollen Zone SB2. 
TA-1402. Karuniidu

Fen peat from depth $400-410 \mathrm{~cm}$. Pollen Zone SB1.

TA-1403. Karuniidu

Bryales peat from depth $450-460 \mathrm{~cm}$. Pollen Zone SB1.

TA-1404. Karuniidu

$4120 \pm 70$

Forest peat from depth $500-510 \mathrm{~cm}$. Beginning of climatic period SB.

TA-1405. Karuniidu

$4300 \pm 70$

Forest peat from depth $520-530 \mathrm{~cm}$. Boundary of Atlantic and Subboreal (AT and SB) climatic periods.

TA-1406. Karuniidu

$6000 \pm 80$

Basal layer of forest peat on sand from depth $530-550 \mathrm{~cm}$. Climatic period AT.

TA-1277. Karuniidu

$1830 \pm 60$

Buried oak trunk found overlying sediments (while digging pond). Date is from 10 outer tree rings. Sample subm 1979 by U Riispere, Inst Zoology and Botany, Tartu, Estonian SSR.

TA-1598. Tuuljärv $380 \pm 100$

Carex-Sphagnum peat from depth $100-105 \mathrm{~cm}$. Pollen Zone SA3.

TA-1599. Tuuljärv $1670 \pm 70$

Forest-Phragmites peat from depth $140-150 \mathrm{~cm}$. Pollen Zone SA2.

TA-1610. Tuuljärv

$2600 \pm 80$

Equisetum-Bryales peat with remains of trees from depth $240-250 \mathrm{~cm}$. Beginning of Pollen Zone SA1.

TA-1613. Tuuljärv

$3040 \pm 100$

Forest peat from depth $320-330 \mathrm{~cm}$. Pollen Zone SB2.

TA-1614. Tuuljärv

$3560 \pm 80$

Forest peat from depth $360-370 \mathrm{~cm}$. Pollen Zone SB2.

TA-1617. Tuuljärv

$4070 \pm 70$

Forest-Phragmites peat from depth $450-460 \mathrm{~cm}$. Pollen Zone SB1.

TA-1615. Tuuljärv

$6560 \pm 90$

Sapropel with remains Equisetum from depth $480-490 \mathrm{~cm}$. Pollen Zone AT2.

TA-1616. Tuuljärv

$7940 \pm 80$

Phragmites-Bryales peat from depth $490-500 \mathrm{~cm}$. End of climatic period BO.

TA-1515. Tuuljärv

$8790 \pm 80$

Partially decomposed Bryales peat from depth $560-570 \mathrm{~cm}$. Beginning of climatic period $\mathrm{BO}$.

TA-1694. Vaskna

$830 \pm 60$

Carex peat with remains of trees from depth $25-30 \mathrm{~cm}$. Pollen Zone SA3. 
TA-1693. Vaskna

$1510 \pm 70$

Forest-Carex peat from depth $30-35 \mathrm{~cm}$. Boundary of Pollen Zones SA2 and SA3.

TA-1692. Vaskna

$2120 \pm 60$

Forest-Carex peat from depth $45-50 \mathrm{~cm}$. Pollen Zone SA2.

TA-1691. Vaskna

$2300 \pm 60$

Forest-Phragmites peat from depth $50-55 \mathrm{~cm}$. Boundary of Pollen Zones SA2 and SA1.

TA-1690. Vaskna

$2590 \pm 60$

Forest-Carex peat from depth $55-60 \mathrm{~cm}$. Boundary of climatic periods SB and SA.

TA-1698. Vaskna

$4210 \pm 60$

Forest-Carex peat from depth $70-75 \mathrm{~cm}$. Pollen Zone SB2.

TA-1688. Vaskna

$\mathbf{5 0 3 0} \pm \mathbf{6 0}$

Forest-Carex peat from depth $80-85 \mathrm{~cm}$. Pollen Zone SB1.

TA-1687. Vaskna

$6850 \pm 60$

Forest-Bryales peat from depth $95-100 \mathrm{~cm}$. Climatic period AT.

TA-1686. Vaskna

$7250 \pm 60$

Equisetum-Eriophorum peat from depth 110-115cm. End of climatic period BO2.

TA-1727. Vaskna

$8050 \pm 60$

Bryales peat from depth $115-120 \mathrm{~cm}$. Pollen Zone BO2.

TA-1685. Vaskna

$8280 \pm 60$

Bryales-Phragmites peat from depth $125-130 \mathrm{~cm}$. Pollen Zone BO2.

TA-1737. Vaskna

$8730 \pm 60$

Bryales peat from depth $145-150 \mathrm{~cm}$. End of climatic period PB.

TA-1684. Vaskna

$9680 \pm 70$

Bryales-Phragmites peat from depth 160-165cm. Upper layer of climatic period PB.

TA-1683. Vaskna

$9870 \pm 70$

Dy from depth $170-175 \mathrm{~cm}$. Climatic period PB.

TA-1682. Vaskna

$9970 \pm 70$

Sapropel from depth $185-190 \mathrm{~cm}$. Climatic period PB.

TA-1600. Vaskna

$9930 \pm 70$

Sapropel from depth 200-205cm. Beginning of climatic period PB.

Järvesoo series

Järvesoo bog, $90 \mathrm{ha}$, lies in Saaremaa Island, 18km W of Kuressaare and is formed of fen and mesotrophic peat. Samples coll 1977 by E Ilves and A Sarv, Inst Zoology and Botany, Inst Geology, Tartu.

TA-771. Järvesoo

$1420 \pm 70$

Peat from depth $20-30 \mathrm{~cm}$. 
TA-772. Järvesoo

$1460 \pm 70$

Peat from depth $60-70 \mathrm{~cm}$.

TA-773. Järvesoo

$1450 \pm 70$

Peat from depth $70-80 \mathrm{~cm}$.

TA-774. Järvesoo

$1750 \pm 70$

Peat from depth $80-90 \mathrm{~cm}$.

TA-775. Järvesoo

$4090 \pm 80$

Peat from depth $100-110 \mathrm{~cm}$.

TA-776. Järvesoo

$4570 \pm 80$

Peat from depth 110-120cm.

TA-778. Järvesoo

$5440 \pm 70$

Peat from depth $130-140 \mathrm{~cm}$.

TA-779A. Järvesoo

$6280 \pm 90$

Peat from depth $160-170 \mathrm{~cm}$.

TA-779B. Järvesoo

$6510 \pm 70$

Lake lime from depth $160-170 \mathrm{~cm}$.

TA-780. Järvesoo

$6770 \pm 90$

Lake lime from depth $220-230 \mathrm{~cm}$.

TA-777. Järvesoo

$7610 \pm 80$

Lake lime from depth $260-270 \mathrm{~cm}$.

TA-781. Järvesoo

$8380 \pm 80$

Lake lime from depth $320-330 \mathrm{~cm}$.

TA-782. Järvesoo

$8800 \pm 90$

Lake lime from depth $340-350 \mathrm{~cm}$.

\section{Ukrainian SSR}

\section{Stojanov series}

Stojanov bog lies in Malye Polesje near Stojanov village, Lvov oblast, Ukrainian SSR. Bog is drained and peat extracted. Peat stratum reaches thickness of $5.25 \mathrm{~m}$. Samples coll and subm 1978 by A T Artjushenko and L G Bezus'ko, N G Holodnyi Inst Botany, Kiev, Ukrainian SSR (Bezus'ko, Ilves \& Kayutina 1980).

TA-1214. Stojanov

$600 \pm 70$

Carex peat with remains of Phragmites from depth $35-40 \mathrm{~cm}$. Pollen Zone SA2.

TA-1215. Stojanov

$1130 \pm 60$

Carex peat with remains of Phragmites from depth $85-90 \mathrm{~cm}$. Boundary of Pollen Zones SA2 and SA1.

TA-1216. Stojanov

$1900 \pm 70$

Carex peat with remains of Phragmites from depth $140-145 \mathrm{~cm}$. Pollen Zone SA1. 
TA-1321. Stojanov

$2680 \pm 70$

Carex-Phragmites peat from depth 202-203cm. Climatic period SB.

TA-1322. Stojanov

$3390 \pm 70$

Carex-Phragmites peat from depth $298-303 \mathrm{~cm}$. Pollen Zone AT2.

TA-1323. Stojanov

$4850 \pm 70$

Carex-Phragmites peat from depth $348-353 \mathrm{~cm}$. Pollen Zone AT2.

TA-1217. Stojanov

$6280 \pm 70$

Carex-Phragmites peat from depth $423-428 \mathrm{~cm}$. Pollen Zone AT1.

TA-1218. Stojanov

$7360 \pm 70$

Phragmites peat from depth $498-503 \mathrm{~cm}$. Pollen Zone SB.

Byelorussian SSR

\section{Sudoble series}

Samples from basal sediments, $300 \mathrm{~m}$ from $\mathrm{W}$ shore of Sudoble Lake, $5 \mathrm{~km}$ SE of Zhodino, Smolevitschki dist, Minsk Obl. Samples coll and subm 1979 by I I Bogdel, V I Lenin Byelorussian State Univ, Minsk (Bogdel et al 1983).

TA-1219. Sudoble

$2360 \pm 80$

Dy from depth $140-160 \mathrm{~cm}$. End of climatic period SB.

TA-1220. Sudoble

$3930 \pm 80$

Dy from depth $280-300 \mathrm{~cm}$. Climatic period SB.

TA-1221. Sudoble

$4960 \pm 70$

Dy from depth $380-400 \mathrm{~cm}$. Beginning of climatic period SB.

TA-1222. Sudoble

$5950 \pm 80$

Dy from depth $485-500 \mathrm{~cm}$. Climatic period AT.

TA-1223. Sudoble

$8510 \pm 70$

Dy from depth $580-600 \mathrm{~cm}$. Boundary of climatic periods BO and AT.

TA-1224. Sudoble

$9080 \pm 90$

Sapropel from depth $680-700 \mathrm{~cm}$. Basal layers of climatic period BO.

TA-1225. Sudoble

$11,160 \pm 100$ AL.

Sapropel with slight admixture of lake lime from depth $770-790 \mathrm{~cm}$. Climatic period

TA-1226. Sudoble

$\mathbf{1 1 , 5 5 0} \pm \mathbf{1 0 0}$

Peat with admixtures of lake lime and macroparticles of wood from depth $875-885 \mathrm{~cm}$. Climatic period AL.

\section{Kobuzi series}

Kobuzi bog lies in Vilei dist, $400 \mathrm{~m} \mathrm{~N}$ of Kobuzi village. Samples coll and subm 1979 by I I Bogdel.

TA-1369. Kobuzi

$730 \pm 60$

Peat with undecayed vegetal remains from depth $22-28 \mathrm{~cm}$. 
TA-1368. Kobuzi

Same type, from depth $28-35 \mathrm{~cm}$.

TA-1367. Kobuzi

$2750 \pm 70$

Same type, from depth $50-55 \mathrm{~cm}$.

TA-1366. Kobuzi

$4320 \pm 70$

Same type, from depth $75-80 \mathrm{~cm}$.

TA-1365. Kobuzi

$6050 \pm 60$

Same type, from depth $100-105 \mathrm{~cm}$.

TA-1364. Kobuzi

$7650 \pm 70$

Peat from depth 125-130cm. Color of peat turns into light brown.

TA-1331. Kobuzi

$8760 \pm 70$

Peat from depth $150-155 \mathrm{~cm}$. Color of peat turns into yellowish brown.

TA-1330. Kobuzi

$8730 \pm 70$

Peat from depth $175-180 \mathrm{~cm}$.

TA-1329. Kobuzi

$9640 \pm 90$

Peat with slightly decayed woody remains from depth $225-230 \mathrm{~cm}$.

TA-1326. Kobuzi

$9550 \pm 80$

Wood "A" from depth $240 \mathrm{~cm}$.

TA-1327. Kobuzi

$10,430 \pm 90$

Wood "B" from depth $240 \mathrm{~cm}$, from layer where partly decayed wood from trunks, roots and branches were collected.

\section{TA-1328. Kobuzi}

$9530 \pm 80$

Forest peat from depth $250-255 \mathrm{~cm}$.

TA-1325. Kobuzi

$10,430 \pm 90$

Forest peat from depth $275-280 \mathrm{~cm}$.

TA-1324. Kobuzi

$11,200 \pm 90$

Peat with sand from depth $295-300 \mathrm{~cm}$.

Georgian SSR

\section{Anaklija series}

Dates of lake and bog sediments reported below refer to complex study of Inguri River basin, W Georgia. Samples coll and subm 1981 by L R Serebryannyi, Inst Geography, Tartu (Serebryannyi et al 1984).

TA-1303. Anaklija $3090 \pm 100$

Peat from depth $217-220 \mathrm{~cm}$. Base of Pollen Zone SB2.

TA-1301. Anaklija $4090 \pm 90$

Peat from depth $315-320 \mathrm{~cm}$. Pollen Zone SB1. 
TA-1300. Anaklija

Peat from depth $380-385 \mathrm{~cm}$. Pollen Zone SB1.

TA-1299. Anaklija

$4570 \pm 70$

Peat from depth $530-535 \mathrm{~cm}$. Pollen Zone AT2.

TA-1298. Anaklija

$5640 \pm 100$

Peat from depth 625-632cm. Pollen Zone AT2.

\section{Hungarian $P R$}

\section{Lovaszberen}

Sample from buried soil from Lovaszberen loess, Hungary, site with coordinates $\left(47^{\circ} 18^{\prime} \mathrm{N}, 18^{\circ} 33^{\prime} \mathrm{E}\right)$. In 1977 while examining the section, numerous large charcoal fragments were observed (Ilves, Pecsi \& Serebryannyi 1980). Sample subm 1977 by M Pecsi, Hungarian Acad Sciences.

\section{TA-1196. Lovaszberen}

$20,220 \pm 300$

\section{REFERENCES}

Bezus'ko, LG, Ilves, EO and Kayutina, TI 1980 New data on vegetation of Maloye Polesje in the Holocene. Ukr Bot Zh 37(5):35-39 (in Russian).

Bogdel, II, Vlassov, BP, Ilves, EO and Klimanov, VA 1983 Sudoble section-A stratotype reconstruction of paleogeographic conditions of the Holocene in central Byelorussia, in History of lakes in the USSR. Abs,
All-Union Mtg, 6th, Tallinn:30-33 (in Russian).

Ilves, E and Mäemets, H 1987 Results of radiocarbon and palynological analyses of coastal deposits of lakes Tuuljärv and Vaskna,-_Palaeohydrology of the temperate zone. Mires and lakes, Tallinn III:108-130.

Ilves, E, Pecsi, M and Serebryannyi, L 1980 Absolute age of buried soil from Lovaszberen loess section. Izv Akad Nauk Est SSR Khim Geol 29(3):118-120 (in Russian).

Mäemets, H 1983 Palynological and radiocarbon data on postglacial vegetational history of Haanja Heights (ESSR). Man, vegetation and soil, Tallinn:98-111.

Serebryannyi, LR, Margalitadze, NA, Janelidze, Ch P, Gey, NA, Ilves, EO, Skobeva, EI, Vishnevskaja, EM, Orlov, AV 1984 Late- and postglacial history of phytolandscapes of Colchis. Proc Acad Sci Georgian SSR Biol Ser 10(5):306-312 (in Russian). 